\title{
Medium Induced Color Switching of a Spiropyran Photochromic Dye: Experimental Studies and Quantum Chemical Calculations
}

\author{
Sarah N. A. Mobarez, Mohamed S. Attia, Mohamed M. Abo-Aly and Mohamed S. A. Abdel- \\ Mottaleb* \\ Nano-Photochemistry and Solar-chemistry Labs, Department of Chemistry, Faculty of Science, Ain Shams University, \\ 11566 Abbassia, Cairo, Egypt,
}

\section{A RT ICLE INFO}

Article history:

Received 04 August 2015

Accepted 12 September 2015

Keywords:

Spiropyran;

Photochromism;

DFT;

Color switching.

\begin{abstract}
A B S T R A C T
The UVA light excitation induced opening of the colorless 2H-1-benzopyran ring of the spiropyran derivative under investigation strongly depends on the polarity of the medium. The molecular skeleton combines a $2 \mathrm{H}$-indole and a 6-nitro,8methoxy benzopyran through a spiro center. Optimized geometry obtained by DFT computations shows that the two fragments are orthogonal to each other and, hence, do not communicate electronically in the ground state of the closed form (SP). The photoinduced ring-opening process generates a planar colored zwitterionic isomer, incorporating a $2 \mathrm{H}$-indolium cation and a 6-nitro,8-methoxy phenolate anion. The photogenerated merocyanine colored isomer reverts thermally to the original species with first-order kinetics. In fact, a complete switching cycle with this photochromic compound can be completed on a second timescale in case of protic polar solvents.
\end{abstract}

\section{Introduction}

The phenomenon of photochromism denotes a special case of a photochemical switchable reaction where a reversible molecular transformation takes place between two geometrical isomers with a significant change in the light absorption window upon exposure to electromagnetic radiation [1]. The photochromic properties of spirooxazines and spiropyrans are determined by the existence and mutual transitions of a closed colorless spiro-form and an open colored merocyanine form. In the closed form, the $\pi$-systems of the two parts of the molecule are mutually perpendicular and have no conjugated bond. Therefore, this form absorbs in the UV region. In the open form, spiropyrans and spirooxazines display a flat geometry and a single $\pi$ system, which gives rise to a strong absorption band in the red spectral region ${ }^{[2]}$. Nitrospiropyrans are members of one of the most popular families of photochromic compounds ${ }^{[3]}$. Irradiation in the UV region leads to the cleavage of the Cspiro $-\mathrm{O}$ bond resulting in cismerocyanine (cis-MC) then the rotation about the central C-C bonds yields trans-MC (Scheme 1). The MC product is a hybrid of zwitterionic and quinoidal resonance forms (Scheme 2 ). The $\mathrm{MC} \rightarrow \mathrm{SP}$ reverse isomerization usually occurs spontaneously and can be accelerated thermally or by visible light ${ }^{[4]}$. Spiropyrans

\footnotetext{
* Corresponding author.

E-mail address: Solar@photoenergy.org
}

are also capable of isomerizing in the presence of metal ions even under dark conditions, since the merocyanine form is stabilized by coordination to the metal ions ${ }^{[5]}$. Spiropyrans belong to a series of photochromic compounds that can be potentially applied in information-recording systems, such as optical memory and switching devices, and have been the most extensively studied for several decades [6]. Many chemosensors based on photochromic spiropyrans allow the real-time monitoring of metal ions in environmental and biological objects ${ }^{[7]}$. Several examples of photochromic systems are sensitive to a number of biological (macro) molecules such as amino acids, proteins, and DNA ${ }^{[8]}$. Solvatochromism is meaningful property of spiropyrans. It has been verified experimentally that only those molecules with $\pi$-electron conjugated system, such as MC, can exhibit solvatochromism. Hence, solvatochromism is a sensitive property of the ring-open $\mathrm{MC}$ form. It is clear that various intermolecular interactions are involved in solvatochromism, such as dispersion force, hydrogen bonding, and polarity, etc. and many parameters or scales have been suggested to estimate the solvents effects on solvatochromism ${ }^{[9]}$. The main problem of spiropyrans is their low stability and for this reason several attempts have been performed in order to graft them in the backbone of a macromolecular chain or to incorporate them into a polymer matrix ${ }^{[10]}$. Attachment 
of spiropyran to inorganic or macromolecular carriers allows one to take advantage of the desired mechanical properties, film formation capabilities, etc., imparted by the carrier and also will increase its resistance to "fatigue", (gradual degradation with increasing number of switching cycles) ${ }^{[4]}$.

So in this article we report on the photochromic behavior of (1',3'-Dihydro-8-methoxy-1',3',3'-trimethyl6-nitrospiro[2H-1-benzopyran-2,2'-(2H)-indole]) (SP) in different polar and non polar environments as protic polar solvents, aprotic polar solvents, non polar solvents, and a mixed solvents system. The solvent polarity effects on the kinetics of the UV irradiation/dark reactions are studied. Furthermore, we used Gaussian 09 quantum chemical package to model the HOMO-LUMO distributions, charge densities, the UV-VIS electronic spectra of the optimized geometry of the open and closed dye forms in the ground and excited states.<smiles>COc1cc([N+](=O)[O-])cc2c1OC1(C=C2)N(C)c2ccccc2C1(C)C</smiles>

$\mathrm{h} \nu$ (UV light)

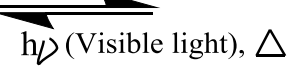<smiles>COC1=CC([N+](=O)[O-])=CC(=CC=C2N(C)c3ccccc3C2(C)C)C1=O</smiles><smiles>COC1=C/C(=C/C=C2\N(C)c3ccccc3C2(C)C)C(=O)C([N+](=O)[O-])=C1</smiles>

Scheme 1: Closed and Open Forms of Spiropyran
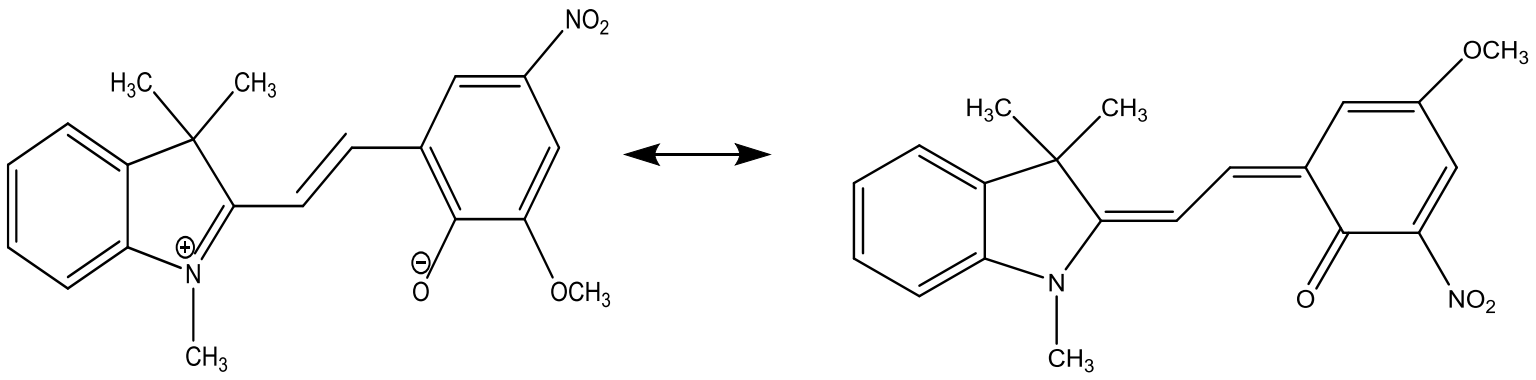

Scheme 2: Resonance in Zwitterionic and Quinoidal MC Forms

\section{Materials and Methods}

\section{Materials}

The spiropyran (SP) (1',3'-Dihydro-8-methoxy-1',3',3'trimethyl-6-nitrospiro[2H-1-benzopyran-2,2'-(2H)-

indole]) (Sigma-Aldrich,97\%) was used as received. Pure grade (Aldrich) solvents were used.

\section{Preparation of Spiropyran solutions}

All the solutions were prepared by taking $0.1 \mathrm{~mL}$ from the stock solution and the solution was completed to $10 \mathrm{~mL}$ by the used solvent. (The typical final SP con centration was $5 \times 10^{-5} \mathrm{M}$ ).

\section{Instruments}

UV-Vis absorption spectra were measured in the range of 250-800 $\mathrm{nm}$ using diode array Ocean optics Spectrometer with spectra suite operating software. The source of UV irradiation is a homemade photoirradiation apparatus with an 8-Watt UVA lamp.

\section{General procedure for the SP photochemical} reactions

The color developing reaction is carried out using 8 watt UVA lamp. The back reaction occurs in the dark at room temperature. The UV-Vis absorption spectra were 
recorded by an ocean optics Spectrophotometer. The photochemical reactions occurred in solutions were carried out in a quartz cell. The cell was exposed to UVA light and the absorbance was measured after appropriate time intervals until the color of the solution was fully developed, and maximum merocyanine concentration was reached (until no change in the photochromic absorbance was observed). Then the back dark reaction was monitored after the light removal using the repeat scan mode. All the measurements were carried out at room temperature $\approx 22^{\circ} \mathrm{C}$.

\section{Methods of data analysis}

\section{Kinetics measurements}

The kinetic rate parameters of the color developing and the back reactions were determined by following the appearance and the disappearance of the colored MC form at its maximum wavelength. The color developing reaction and the back reaction of SP follows the first order kinetics. The integrated form of the first-order rate law for the photocoloration process is given as:

$\ln \left(\left[\mathrm{A}_{\mathrm{MC}}\right]_{\mathrm{t}}-\left[\mathrm{A}_{\mathrm{MC}}\right]_{\text {eq. }}\right)=-\mathrm{k}_{\text {obs }} \mathrm{t}+\ln \left(\left[\mathrm{A}_{\mathrm{MC}}\right]_{\mathrm{o}}-\left[\mathrm{A}_{\mathrm{MC}}\right]_{\text {eq. }}\right)$ where $k_{\text {obs }}$ is the rate constant, $\left[\mathrm{A}_{\mathrm{MC}}\right]_{\text {eq. }},\left[\mathrm{A}_{\mathrm{MC}}\right]_{\mathrm{t}}$ and $\left[\mathrm{A}_{\mathrm{MC}}\right]_{\mathrm{o}}$ are the absorbance of the MC at infinite time, at time $t$, and at zero time, respectively ${ }^{[6]}$. The plot of $\ln \left(\left[\mathrm{A}_{\mathrm{MC}}\right]_{\mathrm{t}}-\left[\mathrm{A}_{\mathrm{MC}}\right]_{\text {eq }}\right)$ against the irradiation time gives a straight line with slope equals $\mathrm{k}_{\mathrm{obs}}$.

The first order rate constants of the decoloration reactions were obtained from the slope of the plot of $\ln \left[\mathrm{A}_{\mathrm{MC}}\right]_{\mathrm{t}}$ against the relaxation time.

\section{Theoretical calculations}

The Theoretical calculations are carried out using Gaussian 09, Gaussian Inc. (USA), quantum chemical package within the framework of density functional theory (DFT) and visualized by Gauss View 05 program. The geometry optimization of closed and open forms of the photochromic dye was carried out in vacuum as implemented in the Gaussian 09 package ${ }^{[11]}$. The geometry of the dye was optimized using the hybrid exchange-correlation B3LYP ${ }^{[11]}$ functional with the 621G-basis set.

\section{Results and Discussion \\ The Solvent effect}

Photochromism is the reversible transformation in a single chemical species between two forms with different absorption spectra. When the colored MC form is the less stable form this is called positive photochromism, otherwise it is called a negative or inverse photochromism. The positive or negative photochromism of spiropyran may depend on the type of the solvent. The spiropyran solutions in non-polar solvents like toluene and 1,4-dioxane show a positive photochromic behavior. The more stable state is the closed spiropyran form and their solutions are colorless in the dark. On the contrary, the spiropyran solutions in polar solvents like ethanol and DMSO show a negative photochromic behavior. The more stable state is the open merocyanine form and their solutions are colored in the dark.
The polar solvents favors the MC formation in the dark and at room temperature and the non-polar solvents promote the SP stabilization. The half-life time of the $\mathrm{MC}$ form, which is an indication for the MC stability, is found to be $160 \mathrm{sec}$ in ethanol, $80 \mathrm{sec}$ in DMF and $8 \mathrm{sec}$ in n-hexane. Ethanol is an example for the protic polar solvents, DMF is an example for aprotic polar solvents and n-Hexane is an example for non-polar solvents. The ring closure reaction $(\mathrm{MC} \rightarrow \mathrm{SP})$ in ethanol is 36 times slower than that in $\mathrm{n}$-hexane. The ethanol solvent highly stabilizes the polar merocyanine form because of the specific interaction due to H-bonding formation. Compared with other solvents (see table 1), the hydrogen bond acceptor ability ( $\beta$-value) of alcohols is relatively higher. The hypsochromic shift observed by increasing solvent polarity indicates a better stabilization of the ground state of the merocyanine form relative to the excited state. So, the ground state has the zwitterionic character, which has a higher electric dipole moment due to the charge separation while the excited state has the quinoidal structure. $\lambda_{\max }$ of the MC form in ethanol equals $564 \mathrm{~nm}$ while that in cyclohexane equals $600 \mathrm{~nm}$. Therefore, the spiropyran exhibit negative solvatochromism (refer to table 1).

In mixed solvents system of a non polar solvent and a polar solvent (1,4-dioxane and distilled water), it was found that by increasing water percentage from $0 \%$ to $50 \%$ the back reaction slows down and the rate constant decreases from $1.6 \times 10^{-2} \mathrm{sec}^{-1}$ in $100 \%$ dioxane solution to $10^{-4} \mathrm{sec}^{-1}$ in 1:1 (dioxane: water) system (Figure 1). MC stabilization reaches its maximum in the $50 \%$ dioxane $+50 \%$ water system and the half life time of MC was 20 times greater than that in pure dioxane solvent, also the MC peak intensity increased and was blue shifted by increasing the system polarity (Table 2 ).

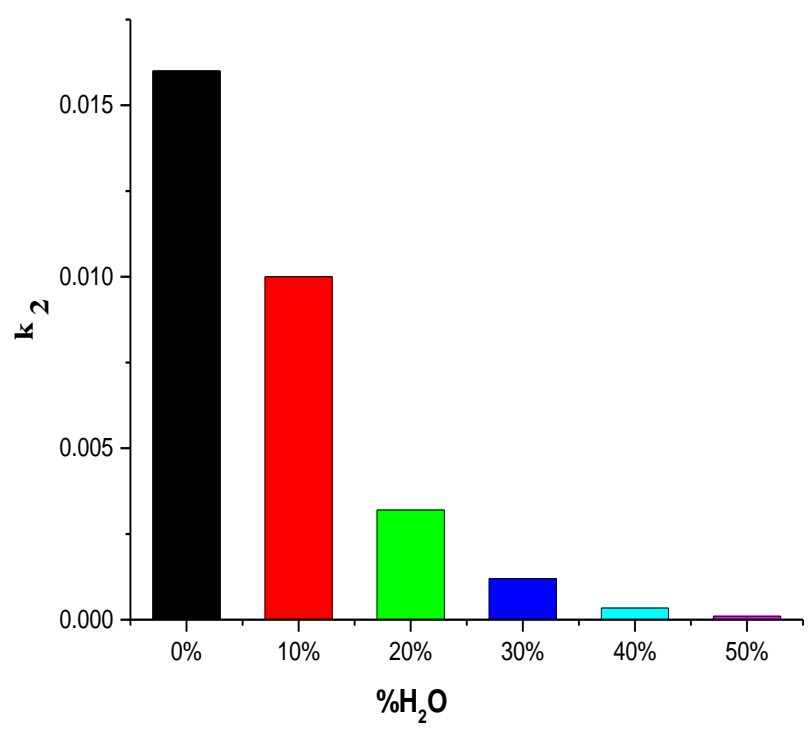

Fig 1: Effect of increasing water percentage in the mixed solvent system of 1,4-dioxane and distilled water on the initial rate constant of the back reaction $\left(\mathrm{k}_{2}\right)$ 
Table 1: The solvent polarity parameters ${ }^{[12-16]}$, MC half life time, initial rate constant of the color developing and back reactions and MC maximum wavelength in different solvents*.

\begin{tabular}{|c|c|c|c|c|c|c|c|c|c|}
\hline Solvent & $\alpha$ & $\boldsymbol{\beta}$ & $\pi$ & $\mathbf{F}(\mathbf{D})$ & $\mathbf{F}(\mathbf{n})$ & $t_{1 / 2}, \sec$ & $\mathrm{k}_{1} \times 10^{3}, \mathrm{sec}^{-1}$ & $\mathrm{k}_{2} \times 10^{3}, \mathrm{sec}^{-1}$ & $\lambda_{\mathrm{MC}}, \mathbf{n m}$ \\
\hline Methanol & 0.93 & 0.77 & 0.6 & 0.477 & 0.169 & 80 & 8.0 & 2.5 & 552 \\
\hline Ethanol & 0.83 & 0.62 & 0.54 & 0.47 & 0.181 & 160 & 12.0 & 2.5 & 564 \\
\hline DMSO & 0 & 0.76 & 1 & 0.485 & 0.221 & 118 & 12.0 & 1.7 & 553 \\
\hline DMF & 0 & 0.76 & 0.88 & 0.48 & 0.204 & 80 & 8.5 & 5.6 & 584 \\
\hline 1,4-Dioxane & 0 & 0.37 & 0.55 & 0.223 & 0.203 & 40 & 24.0 & 16.0 & 604 \\
\hline Benzene & 0 & 0.11 & 0.588 & 0.232 & 0.228 & 35 & 38.0 & 22.0 & 612 \\
\hline Toluene & 0 & 0.1 & 0.535 & 0.241 & 0.226 & 40 & 34.0 & 16.0 & 609 \\
\hline Cyclohexane & 0 & 0 & 0 & 0.2 & 0.204 & 13 & 47.0 & 80.0 & 600 \\
\hline n-Hexane & 0 & $\mathbf{0}$ & -0.08 & 0.188 & 0.186 & 8 & 40.0 & 90.0 & 598 \\
\hline
\end{tabular}

* $\alpha$ is the solvent hydrogen bond -donor ability, $\beta$ is the solvent hydrogen bond - acceptor ability, $\pi$ is the intrinsic solvent polarity. $\mathrm{F}(\mathrm{D})$ is the dielectric constant function, $\mathrm{F}(\mathrm{n})$ is the refractive index function, functions $\mathrm{F}(\mathrm{D}), \mathrm{F}(\mathrm{n})$ are macroscopic solvent polarity parameters. $t_{1 / 2}$ is the half life time of the MC form (the time taken by the MC maximum absorbance to fall to its half value), $\lambda_{\mathrm{MC}}$ is the maximum wavelength of MC. (SP concentration $=5 \times 10^{-5} \mathrm{M}$ ), DMSO (dimethyl sulphoxide), DMF (dimethyl formamide).

Table 2: Effect of increasing water percentage in the mixed solvent system of 1,4-dioxane/water on the initial rate constant of the color developing and back reactions, MC half life time and MC maximum wavelength. (SP concentration $=5 \times 10^{-5} \mathrm{M}$ ).

\begin{tabular}{|c|c|c|c|c|}
\hline$\%$ Dioxane $/ \mathrm{H}_{2} \mathrm{O}$ & $\mathrm{k}_{1} \times 10^{3}, \mathrm{sec}^{-1}$ & $\mathrm{k}_{2} \times 10^{4}, \mathrm{sec}^{-1}$ & $\mathbf{t}_{1 / 2}$, sec & $\lambda_{\mathrm{MC}}, \mathbf{n m}$ \\
\hline $100 / 0$ & 24.0 & 160.0 & 40 & 604 \\
\hline $90 / 10$ & 20.0 & 100.0 & 45 & 573 \\
\hline $80 / 20$ & 7.6 & 32.0 & 110 & 566 \\
\hline $70 / 30$ & 5.2 & 12.0 & 230 & 560 \\
\hline $60 / 40$ & 3.8 & 3.4 & 390 & 555 \\
\hline $50 / 50$ & 2.7 & 1.0 & 800 & 551 \\
\hline
\end{tabular}

$\mathrm{k}_{1}, \mathrm{k}_{2}$ : The rate constants of the color developing and back reactions respectively, $\mathrm{t}_{1 / 2}:$ the half life time of the MC form and $\lambda_{\mathrm{MC}}$ : the maximum wavelength of the MC form.

\section{UV irradiation and relaxation}

Upon UV irradiation, the absorbance of both the MC form and $\mathrm{MC}-\mathrm{M}^{3+}$ complexes increases by increasing the UV irradiation time and the absorbance of the closed SP form decreased (Figures 2, 4 and 6). After the absorbance of the MC form reached its maximum value the relaxation is allowed to take place and the opposite observation happened (Figures 3, 5 and 7). The isosbestic point existed in all the SP spectra in the different environments (polar, nonpolar and mixed solvents). The peak between $350-400 \mathrm{~nm}$ is usually red shifted upon the UV irradiation and blue shifted by relaxation, in addition to that the absorbance of this peak at the start of the ring closure reaction in 1,4-dioxan, toluene and benzene solvents began to decrease and after some time it started to increase again.

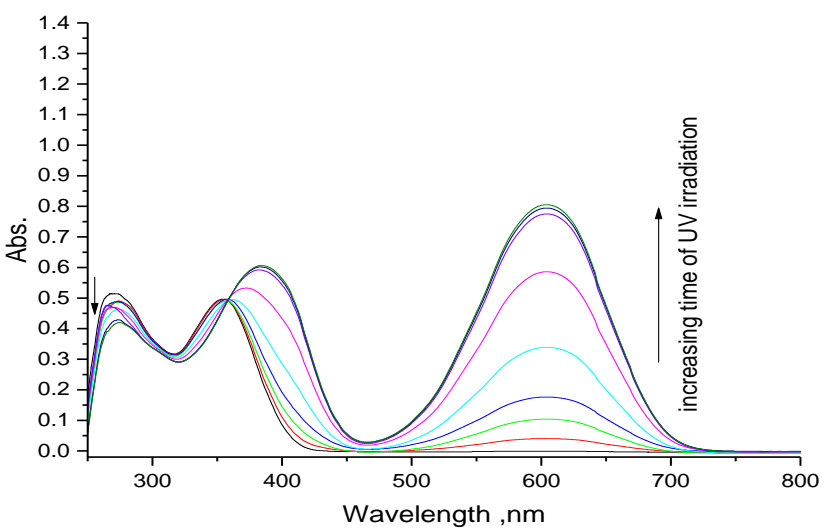

Fig 2: Effect of the time of UV A-irradiation on the absorption spectrum of $5 \times 10^{-5} \mathrm{M}$ of SP in 1,4 Dioxane . 1) zero time, 2 ) 2, 3) 6, 4) 12, 5) 25, 6) 55, 7) 100, 8) 235, 9) $550 \mathrm{sec}$. 


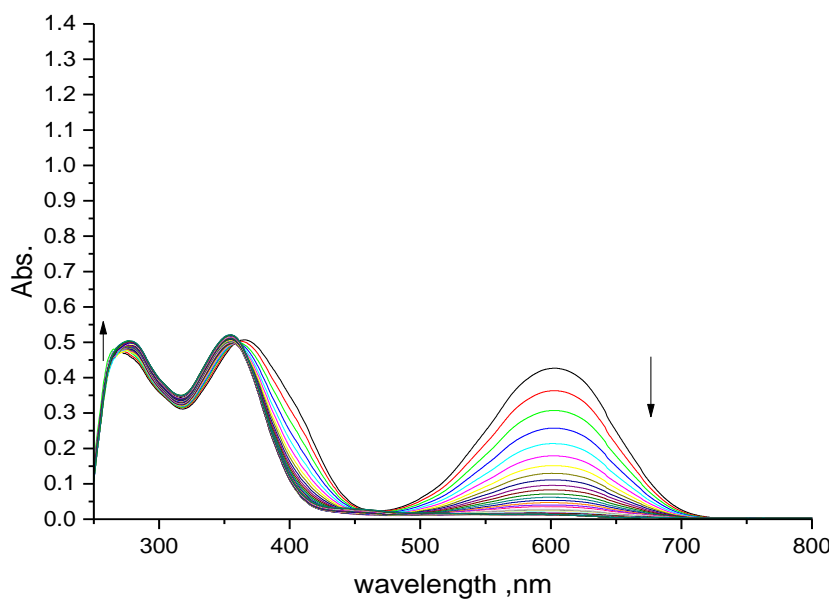

Fig 3: The change in the absorption spectrum of $5 \times 10^{-5} \mathrm{M}$ of SP in 1,4- Dioxane every $10 \mathrm{sec}$ during the dark reaction.

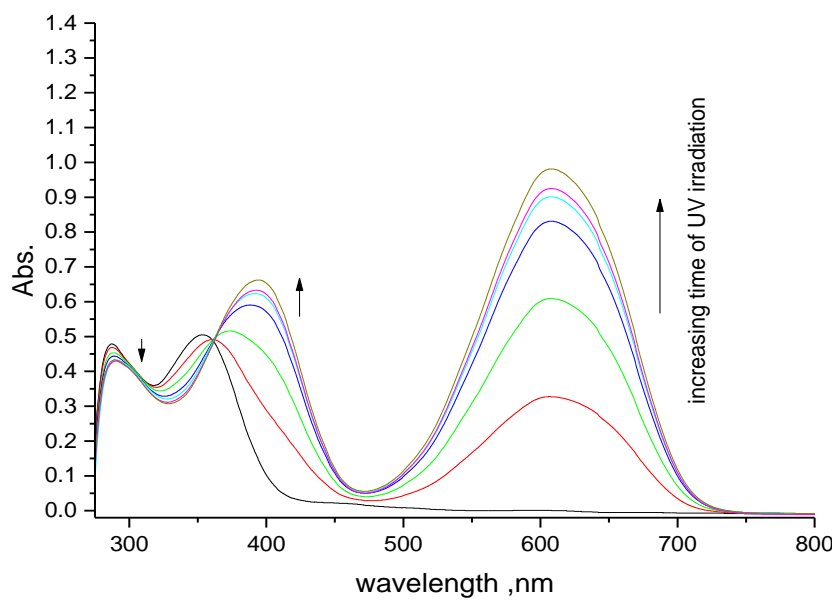

Fig 4: Effect of the time of UV A-irradiation on the absorption spectrum of $5 \times 10^{-5} \mathrm{M}$ of $\mathrm{SP}$ in toluene. 1-6 represent zero time, 10, 25, 55, 100, 160 and $235 \mathrm{sec}$, respectively.

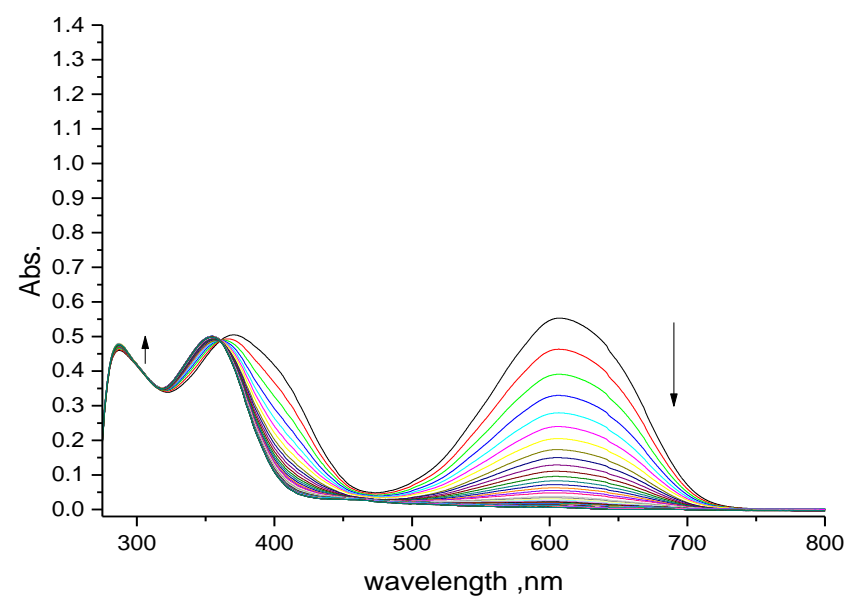

Fig 5: The change in the absorption spectrum of $5 \times 10^{-5} \mathrm{M}$ of SP in toluene every $10 \mathrm{sec}$ during the dark reaction.

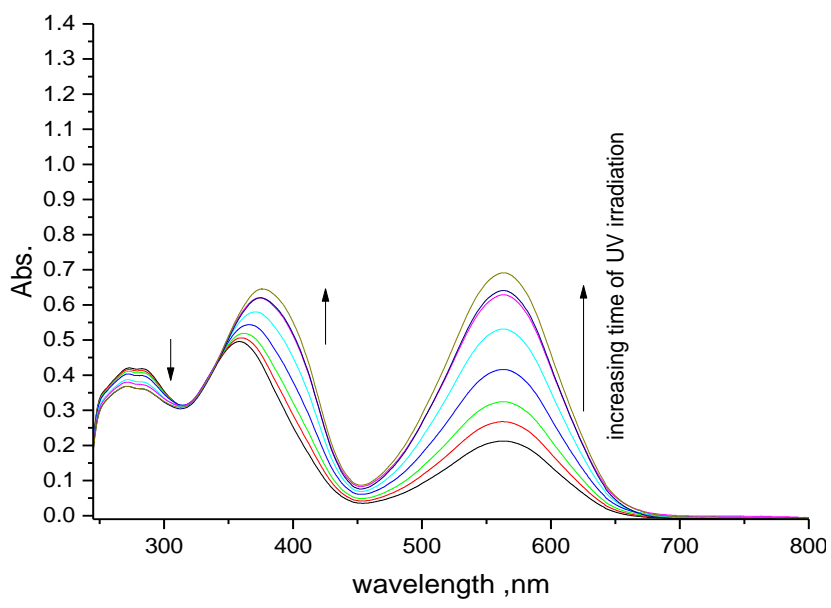

Fig 6: Effect of the time of UV A-irradiation on the absorption spectrum of $5 \times 10^{-5} \mathrm{M}$ of SP in ethanol. 1-6 represent zero time, 10, 25, 55, 100, 160 and $325 \mathrm{sec}$, respectively.

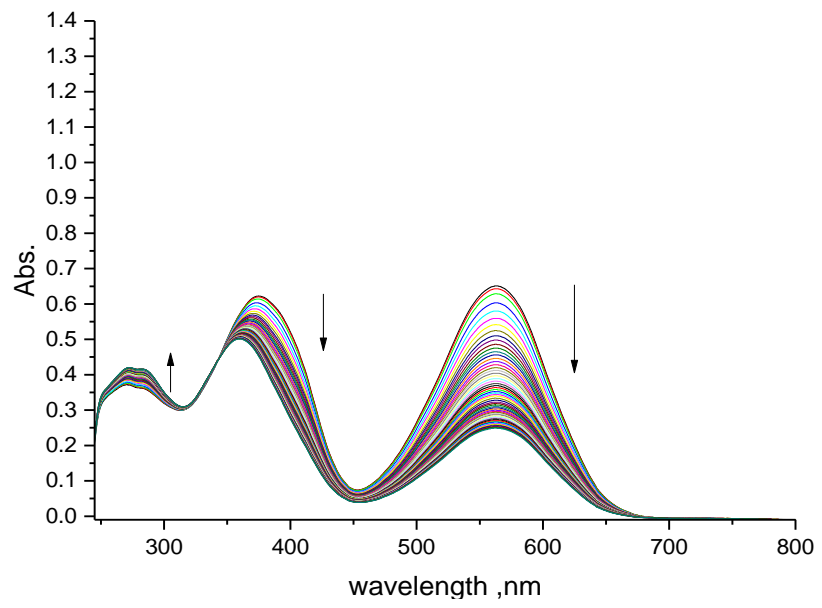

Fig 7: The change in the absorption spectrum of $5 \times 10^{-5} \mathrm{M}$ of $\mathrm{SP}$ in ethanol every $10 \mathrm{sec}$ during the dark reaction.

Molecular structure, charge distribution and the UVVIS electronic spectra of the closed and open forms of the photochromic dye

When the spiropyran was irradiated by UV the cleavage of the $\mathrm{C}_{\text {spiro- }} \mathrm{O}$ bond, which is the less stable part of the molecule, occurred. We studied the molecular geometry of the closed and open forms in gas-phase. Upon the spiropyran-merocyanine transformation the $\mathrm{C}_{7}$ atom $\left(\mathrm{C}_{\text {spiro, }}\right.$, Figure 8) changed from $\mathrm{sp}^{3}$ hybridized to $\mathrm{sp}^{2}$ hybridized (Figure 9) and the two aromatic moieties of the molecule changed from being perpendicular and became planar. DF calculations confirm the charge transfer nature of the electronic transitions.

The electron density in HOMO of the closed SP form is localized on the aromatic moiety linked to the $\mathrm{N}$-methyl group while the electron density in the LUMO is localized on the aromatic moiety linked to the $\mathrm{NO}_{2}$ group (see Figures 10 and 11). The HOMO and LUMO orbitals of MC form are spread over the whole molecular skeleton, (Figures 12 and 13). 


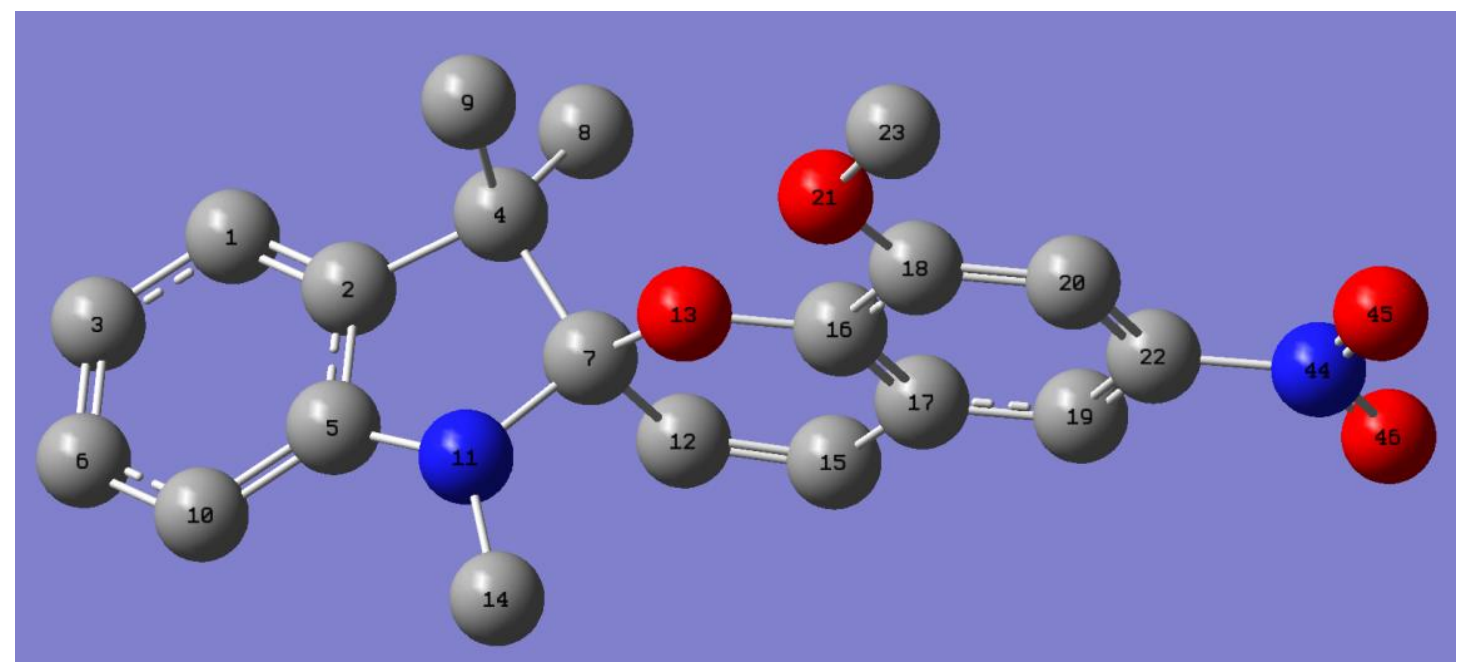

Figure 8: The optimized geometry of the colorless SP structure ( $\mathrm{H}$ atoms are hidden for clarity).

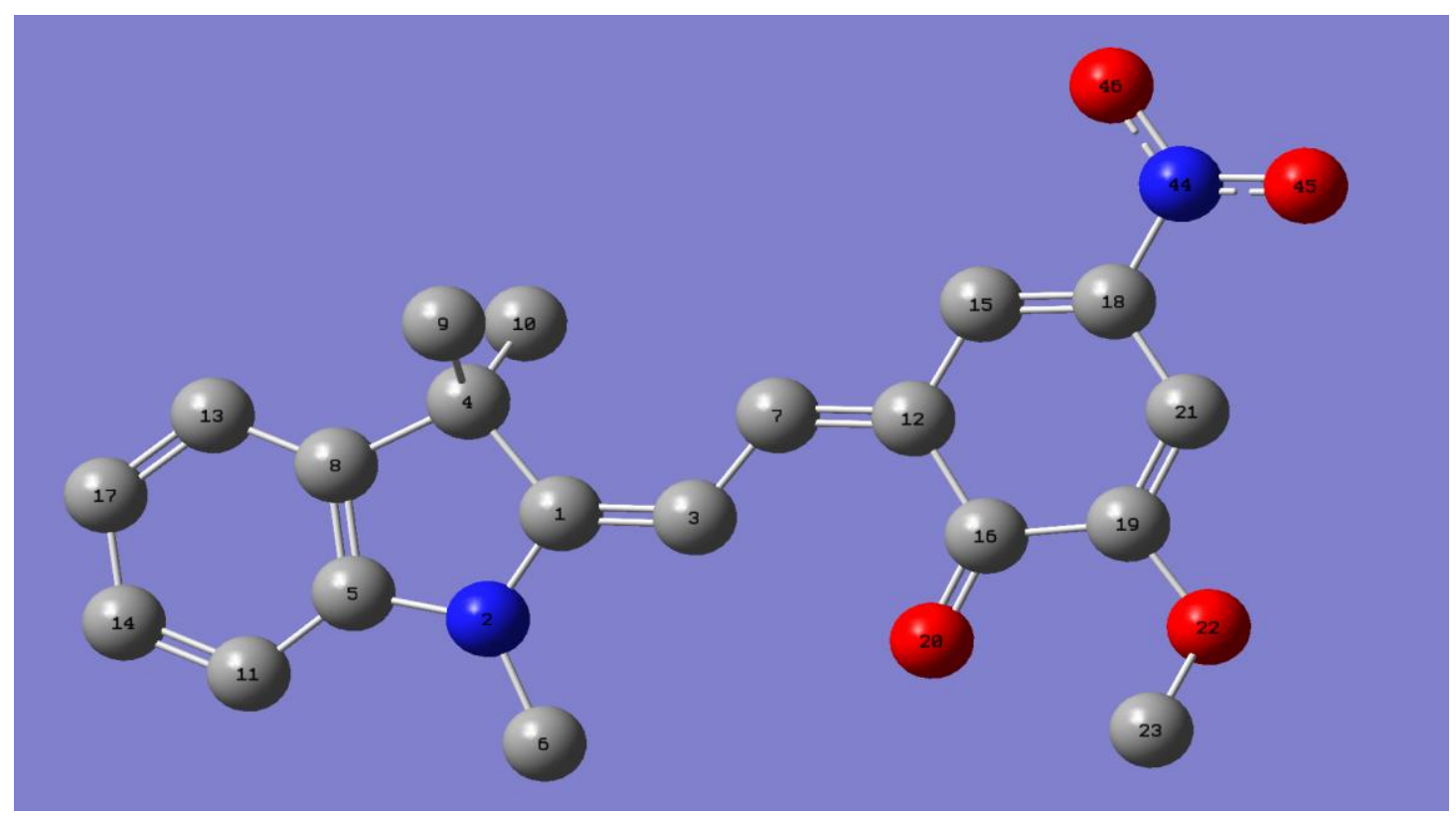

Figure 9: The optimized geometry of the quinoidal structure.

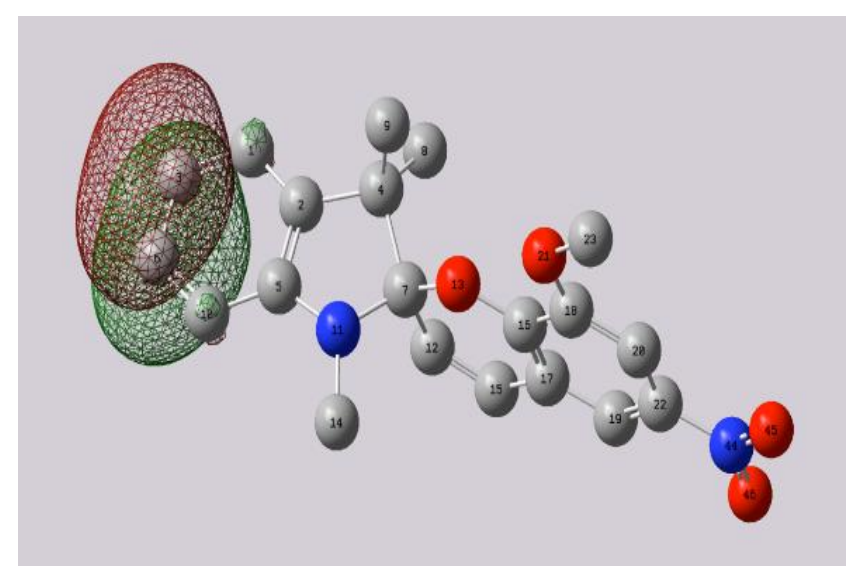

Fig 10: HOMO of SP Form in the ground state.

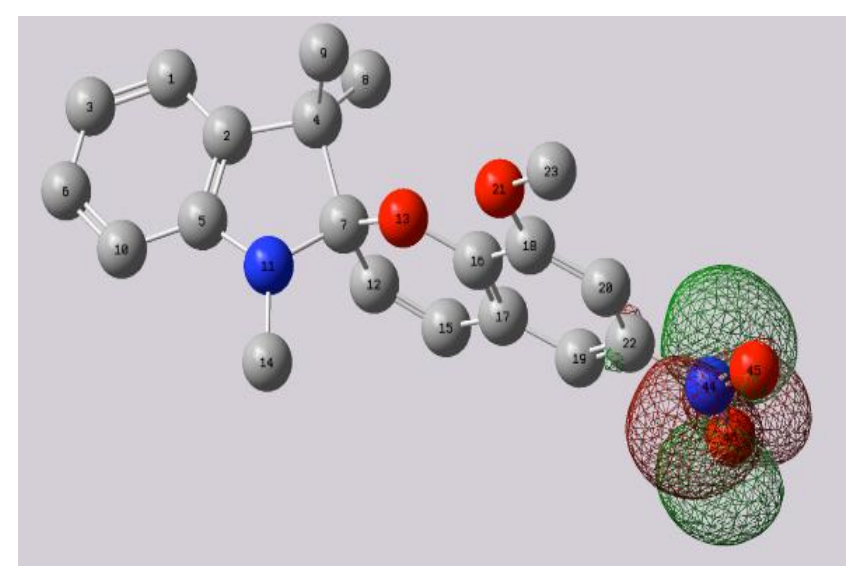

Fig 11: LUMO of SP form in the ground state. 


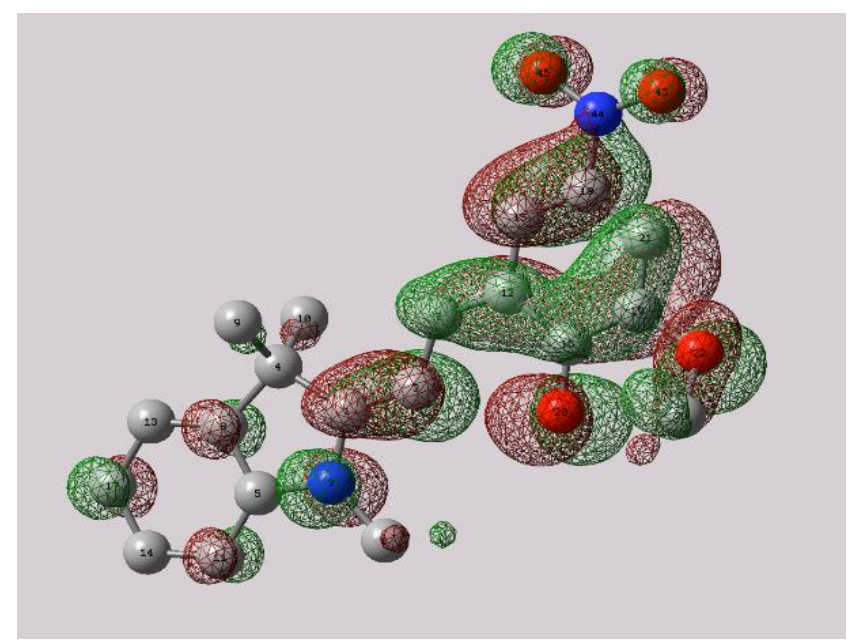

Fig 12: HOMO of MC form of the excited state.

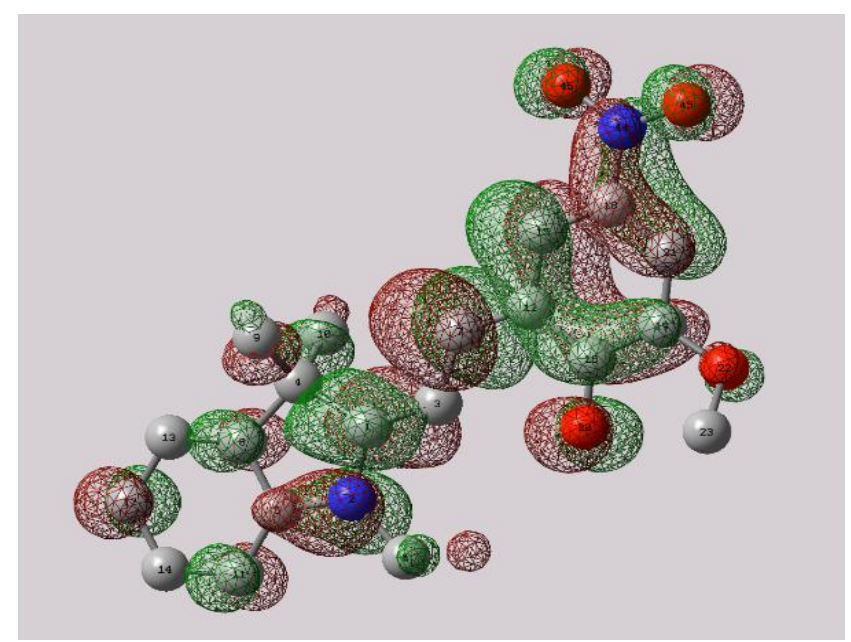

Fig 13: LUMO of MC form in the excited state.

\section{Acknowledgment}

This work was supported by the Nano-Photochemistry and Solarchemistry labs, Department of Chemistry, Faculty of Science, Ain Shams University. Quantum chemical computations were performed by Prof. Dr. M. S. Abdel-Mottaleb.

\section{References}

1) Murugan, N. A., Chakrabarti, S., and Ågren, H. (2011). Solvent dependence of structure, charge distribution, and absorption spectrum in the photochromic merocyanine -spiropyran pair. J. Phys. Chem., 115: 4025-4032.

2) Glebov, E. M., Vorobyev, D. Yu., Grivin, V. P., Plyusnin, V. F., Metelitsa, A.V., Voloshin, N. A., Minkin, V. I., and Micheau, J. C. (2006). Photochemistry of phenanthroline-containing spirooxazines in a low-temperature methanol matrix. Chemical Physics, 323: 490-500.

3) Tomasulo, M., Sortino, S., and Raymo, F. M. (2008). A new family of photochromic compounds based on the photoinduced opening and thermal closing of [1,3]oxazine rings. Journal of Photochemistry and Photobiology A: Chemistry, 200: 44-49.

4) Klajn, R. (2014). Spiropyran-based dynamic materials. Chem. Soc. Rev., 43: 148-184.

5) Yagi, S., Nakamura, S., Watanabe, D., and Nakazumi, H. (2009). Colorimetric sensing of metal ions by bis(spiropyran) podands: Towards naked-eye detection of alkaline earth metal ions. Dyes and Pigments, 80: 98-105.

6) Wu, Y., Sasaki, T., Kazushi, K., Seo, T. and Sakurai, K. (2008). Interactions between spiropyrans and room-temperature ionic liquids: photochromism and solvatochromism. J. Phys. Chem., 112: 7530-7536.

7) Chernyshev, A. V., Voloshin, N. A., Metelitsa, A. V., Tkachev, V. V., Aldoshin, S. M., Solov'eva, E., Rostovtseva, I. A. and Minkin, V. I. (2013). Metal complexes of new photochromic chelator: Structure, stability and photo dissociation. Journal of Photochemistry and Photobiology A: Chemistry, 265: 1-9.

8) Paramonov, S. V., Lokshin, V. and Fedorova, $O$. A. (2011). Spiropyran, chromene or spirooxazine ligands: insights into mutual relations between complexing and photochromic properties. Journal of Photochemistry and Photobiology C: Photochemistry Reviews, 12: 209- 236.

9) Tian, W. and Tian, J. (2014). An insight into the solvent effect on photo-, solvato-chromism of spiropyran through the perspective of intermolecular interactions. Dyes and Pigments, 105: 66-74.

10) Samoladas, A., Bikiaris, D., Zorba, T., Paraskevopoulos, K. M., and Jannakoudakis, A. (2008). Photochromic behavior of spiropyran in polystyrene and polycaprolactone thin films - Effect of UV absorber and antioxidant compound. Dyes and Pigments, 76: 386-393.

11) Frisch, M. J., Trucks, G. W. and Schlegel, H. B. (2013). GAUSSIAN 09, Revision D.01, Gaussian, Wallingford, Conn, USA.

12) Kosower, E. M. (1985). Mechanism of fast intramolecular electron-transfer reactions. J. Am. Chem. Soc., 107: 1114-1118.

13) Sun, M. and Song, P. (1977). Solvent effects on the fluorescent states of indole derivatives-dipole moments. Journal of Photochemistry and Photobiology, 25: 3-9.

14) Koutek, B. (1978). Dipole moments in excited state. Statistical investigation of methods employing solvatochromism. Collect. Czech. Chem. Commun., 43: 2368-2386. 
15) Belletete, M. and Durocher, G. (1982). Electronic spectroscopy of aromatic Schiff bases. V. Specific interactions involved between some typical benzylideneaniline molecules and small polar molecules. Can. J. Chem., 60: 2332-2339.
16) Abdel-Mottaleb, M. S. A., El-Zawawi, F. M., Antonious, M. S., Abo-Aly, M. M., and El-Feky, M. (1989). Photophysical behaviour of a simple merocyanine and its analytical implications. Journal of Photochemistry and Photobiology, A: Chemistry, 46: $99-112$. 